\title{
A Heredity Property of Sufficiency
}

\author{
Jürgen HILLE
}

University of Münster

(Communicated by T. Suzuki)

\begin{abstract}
If $(X, \mathscr{A})$ is a measurable space, $\left(\mathscr{P}_{n}\right)_{n \in \mathrm{N}}$ is an increasing sequence of nonempty sets $\mathscr{P}_{n}$ of probability measures and $\mathscr{B}_{n}$ is a sub- $\sigma$-field of $\mathscr{A}$ which is sufficient for the statistical experiment $\left(X, \mathscr{A}_{1}, \mathscr{P}_{n}\right)$, $n \in \mathbf{N}$, then the terminal $\sigma$-field of the sequence $\left(\mathscr{B}_{n}\right)_{n \in \mathbf{N}}$ contains a $\sigma$-field which is sufficient for $\bigcup_{n \in \mathbf{N}} \mathscr{P}_{n}$.
\end{abstract}

\section{Introduction.}

D. Landers has shown in [4], that the terminal $\sigma$-field $\mathscr{B}_{\infty}$ of a sequence $\left(\mathscr{B}_{n}\right)_{n \in \mathbf{N}}$ of sub- $\sigma$-fields of a given $\sigma$-field $\mathscr{A}$ is minimal sufficient for a class $\mathscr{P}$ of probability measures on $\mathscr{A}$, if $\mathscr{B}_{n}$ is minimal sufficient for $\mathscr{P}_{n}$, where $\mathscr{P}_{n} \subset \mathscr{P}, n \in \mathbf{N}$, are subsets such that $\mathscr{P}_{n} \subset \mathscr{P}_{n+1}, n \in \mathbf{N}$, and $\mathscr{P}=\bigcup_{n \in \mathbf{N}} \mathscr{P}_{n}$. If the assumption of minimality is dropped and $\mathscr{A}$ is countably generated, the terminal $\sigma$-field is sufficient ([4], p. 204, Proposition 9). If $\mathscr{B}_{n}$ is sufficient for $\mathscr{P}_{n}$ but not necessarily minimal sufficient and $\mathscr{A}$ is not countably generated, $\mathscr{B}_{\infty}$ is not sufficient in general ([4], p. 203, Example 8). We show in this paper, that in any case $\mathscr{B}_{\infty}$ contains a sub- $\sigma$-field which is sufficient for $\mathscr{P}$, if $\mathscr{B}_{n}$ is sufficient for $\mathscr{P}_{n}$.

\section{Notations.}

A statistical experiment is a triple $(X, \mathscr{A}, \mathscr{P})$, where $(X, \mathscr{A})$ is a measurable space and $\mathscr{P}$ is a nonempty set of probability measures on $(X, \mathscr{A})$. The system of all $\mathscr{A}$-measurable subsets of $X$, which are $P$-null sets, for all $P \in \mathscr{P}$, is denoted by $\mathscr{N}(\mathscr{P})$. If $\mathscr{E}$ is a system of subsets of $X$, the $\sigma$-field generated by $\mathscr{E}$ is denoted by $S(\mathscr{E})$. If $g, h: X \rightarrow \mathbf{R}$ are $\mathscr{A}$-measurable functions we write $g=h[\mathscr{P}]$ if $P\{x \in X \mid g(x) \neq h(x)\}=0$, for every $P \in \mathscr{P}$. For $\mathscr{A}$-measurable subsets $A_{1}, A_{2}$ of $X$ we write $A_{1}=A_{2}[\mathscr{P}]$ instead of $1_{A_{1}}=1_{A_{2}}[\mathscr{P}]$. If $\mathscr{B}$ and $\mathscr{C}$ are sub- $\sigma$-fields of $\mathscr{A}, \mathscr{B} \subset \mathscr{C}[\mathscr{P}]$ means that for every $B \in \mathscr{B}$ there is a set $C \in \mathscr{C}$ such that $B=C[\mathscr{P}]$. It is easy to see that $S(\mathscr{C} \cup \mathscr{N}(\mathscr{P}))$ consists exactly of those sets $A \in \mathscr{A}$ for which there is a set $C \in \mathscr{C}$ such that $A=C[\mathscr{P}]$. Hence $\mathscr{B} \subset \mathscr{C}[\mathscr{P}]$ is equivalent to $\mathscr{B} \subset S(\mathscr{C} \cup \mathscr{N}(\mathscr{P}))$. A sub- $\sigma$-field $\mathscr{B} \subset \mathscr{A}$ is called sufficient 
for $(X, \mathscr{A}, \mathscr{P})$, if for every bounded $\mathscr{A}$-measurable function $f: X \rightarrow \mathbf{R}$ there is a $\mathscr{B}$-measurable function $g: X \rightarrow \mathbf{R}$ such that $g$ is a version of the conditional expectation $E_{P}(g \mid \mathscr{B})$, for all $P \in \mathscr{P}$.

\section{Results.}

We need three auxiliary results:

LEMMA 1. Let $(X, \mathscr{A})$ be a measurable space, $\mathscr{B} \subset \mathscr{A}$ be a $\sigma$-field and $\left(\mathscr{P}_{n}\right)_{n \in \mathbf{N}}$ be an increasing sequence of nonempty classes of probability measures on $(X, \mathscr{A})$. If $\mathscr{B}$ is sufficient for every statistical experiment $\left(X, \mathscr{A}, \mathscr{P}_{n}\right)$ then it is sufficient for $(X, \mathscr{A}, \mathscr{P})$, where $\mathscr{P}=\bigcup_{n=1}^{\infty} \mathscr{P}_{n}$.

ProOf. [4], p. 206, Lemma 4.

LEMMA 2. Let $(X, \mathscr{A}, \mathscr{P})$ be a statistical experiment and let $\left(\mathscr{B}_{n}\right)_{n \in \mathrm{N}}$ be a sequence of sufficient sub- $\sigma$-fields of $\mathscr{A}$ such that

$$
\mathscr{B}_{n+1} \subset \mathscr{B}_{n} \quad[\mathscr{P}] \quad \forall n \in \mathbf{N} .
$$

Then the terminal $\sigma$-field

$$
\mathscr{B}_{\infty}=\bigcap_{n=1}^{\infty} S\left(\bigcup_{m=n}^{\infty} \mathscr{B}_{m}\right)
$$

is sufficient.

PROOF. Let $\tilde{\mathscr{B}}_{n}:=S\left(\mathscr{B}_{n} \cup \mathscr{N}(\mathscr{P})\right)$, for all $n \in \mathbf{N}$. Then $\tilde{\mathscr{B}}_{n+1} \subset \tilde{\mathscr{B}}_{n}$, for all $n \in \mathbf{N}$, and

$$
\mathscr{B}_{\infty} \subset \tilde{\mathscr{B}}_{\infty}:=\bigcap_{n=1}^{\infty} \tilde{\mathscr{B}}_{n} .
$$

A result of Burkholder ([2], p. 1197, Corollary 2) yields the sufficiency of $\widetilde{\mathscr{B}}_{\infty}$. Hence, if $f$ is a bounded real valued measurable function on $X$, there exists a $\tilde{\mathscr{B}}_{\infty}$-measurable and bounded function $g$ on $X$ which is a version of the conditional expectation $E_{P}\left(f \mid \tilde{\mathscr{B}}_{\infty}\right)$ simultaneously for all $P \in \mathscr{P}$. By definition of $\widetilde{\mathscr{B}}_{\infty}$, for every $n \in \mathbf{N}$ there is a $\mathscr{B}_{n}$-measurable real valued function $g_{n}$ satisfying $g_{n}=g$ [P] (comp. [5], p. 56, Lemma 1.10.3). Since

$$
g=g_{*}:=\limsup _{n \rightarrow \infty} g_{n}[\mathscr{P}] \text {, }
$$

$g_{*}$ is a $\mathscr{B}_{\infty}$-measurable version of $E_{P}\left(f \mid \tilde{\mathscr{B}}_{\infty}\right), P \in \mathscr{P}$, and consequently $g_{*} \in$ $\bigcap_{P \in \mathscr{P}} E_{P}\left(f \mid \mathscr{B}_{\infty}\right)$.

LEMMA 3. Let $(X, \mathscr{A}, \mathscr{P})$ be a statistical experiment and let $\mathscr{B}, \mathscr{C}$ be sub- $\sigma$-fields of $\mathscr{A}$ such that $\mathscr{N}(\mathscr{P}) \subset \mathscr{C}$. Then

$$
\mathscr{C} \cap S(\mathscr{B} \cup \mathscr{N}(\mathscr{P}))=S((\mathscr{C} \cap \mathscr{B}) \cup \mathscr{N}(\mathscr{P}))
$$


ProOF. $\mathscr{C} \cap S(\mathscr{B} \cup \mathscr{N}(\mathscr{P}))$ is a $\sigma$-field which contains $\mathscr{C} \cap \mathscr{B}$ and $\mathscr{N}(\mathscr{P})$. Hence $\mathscr{C} \cap S(\mathscr{B} \cup \mathscr{N}(\mathscr{P})) \supset S((\mathscr{C} \cap \mathscr{B}) \cup \mathscr{N}(\mathscr{P}))$. Conversely let $C$ be an element of $\mathscr{C} \cap$ $S(\mathscr{B} \cup \mathscr{N}(\mathscr{P}))$. Then there is a set $B \in \mathscr{B}$ such that $C=B[\mathscr{P}]$. Hence $C \Delta B \in$ $\mathscr{N}(\mathscr{P}) \subset \mathscr{C}, \Delta$ denoting the symmetric difference, and so $B=C \Delta(C \Delta B)$ is an element of $\mathscr{C} \cap \mathscr{B}$. This shows $C \in S((\mathscr{C} \cap \mathscr{B}) \cup \mathscr{N}(\mathscr{P}))$.

THEOREM 1. Let $(X, \mathscr{A})$ be a measurable space, let, for all $n \in \mathbf{N}, \mathscr{B}_{n}$ be a sub- $\sigma-$ field of $\mathscr{A}$ which is sufficient for the statistical experiment $\left(X, \mathscr{A}, \mathscr{P}_{n}\right)$, where $\left(\mathscr{P}_{n}\right)_{n \in \mathbf{N}}$ is an increasing sequence of nonempty classes of probability measures on $(X, \mathscr{A})$. Let $\mathscr{P}:=\bigcup_{n=1}^{\infty} \mathscr{P}_{n}$. Then the terminal $\sigma$-field $\mathscr{B}_{\infty}:=\bigcap_{n=1}^{\infty} S\left(\bigcup_{m \geq n} \mathscr{B}_{m}\right)$ contains a sub- $\sigma-$ field $\mathscr{B}_{*}$ which is sufficient for $(X, \mathscr{A}, \mathscr{P})$.

Proof. For all $n \in \mathbf{N}, k \geq n$ and $m \geq k$ let

$$
\mathscr{B}(n, k, m):=\bigcap_{i=k}^{m-1} S\left(\mathscr{B}_{i} \cup \mathscr{N}\left(\mathscr{P}_{n}\right)\right) \cap \mathscr{B}_{m}
$$

(the empty intersection is understood to be the $\sigma$-field $\mathscr{A}$, hence $\left.\mathscr{B}(n, k, k)=\mathscr{B}_{k}\right)$. If $i \geq k \geq n$ then $\mathscr{B}_{i}$ is sufficient for $\mathscr{P}_{n}$, since $\mathscr{P}_{n} \subset \mathscr{P}_{i}$. Burkholder (comp. [2], p. 1196, Theorem 4) has proved, that $\mathscr{B}(n, k, m)$ is therefore sufficient for $\mathscr{P}_{n}$. We have

$$
\mathscr{B}(n, k, m+1) \subset \bigcap_{i=k}^{m} S\left(\mathscr{B}_{i} \cup \mathscr{N}\left(\mathscr{P}_{n}\right)\right)=S\left(\mathscr{B}(n, k, m) \cup \mathscr{N}\left(\mathscr{P}_{n}\right)\right),
$$

for all $m \geq k$, where the last equality follows from Lemma 3, applied to the $\sigma$-fields $\mathscr{C}:=\bigcap_{i=k}^{m-1} S\left(\mathscr{B}_{i} \cup \mathscr{N}\left(\mathscr{P}_{n}\right)\right)$ and $\mathscr{B}=\mathscr{B}_{m}$. Hence $(\mathscr{B}(n, k, m))_{m \geq k}$ is a $\mathscr{P}_{n}$-essentially decreasing sequence of sufficient subfields. By Lemma 2 the terminal $\sigma$-field

$$
\mathscr{B}(n, k):=\bigcap_{i=k}^{\infty} S\left(\bigcup_{j \geq i} \mathscr{B}(n, k, j)\right)
$$

is sufficient for $\mathscr{P}_{n}$, for every $k \geq n$. Next observe that $(\mathscr{B}(n, k))_{k \geq n}$ is increasing: By definition one has

$$
\mathscr{B}(n, k, m) \subset \mathscr{B}(n, k+1, m) \quad \forall n \in \mathbf{N}, k \geq n, m \geq k+1,
$$

and consequently

$$
\begin{aligned}
\mathscr{B}(n, k) & =\bigcap_{i=k+1}^{\infty} S\left(\bigcup_{j \geq i} \mathscr{B}(n, k, j)\right) \\
& \subset \bigcap_{i=k+1}^{\infty} S\left(\bigcup_{j \geq i} \mathscr{B}(n, k+1, j)\right)=\mathscr{B}(n, k+1),
\end{aligned}
$$

for all $n \in \mathbf{N}, k \geq n$ (note that $\mathscr{B}(n, k)=\bigcap_{i=l}^{\infty} S\left(\bigcup_{j \geq i} \mathscr{B}(n, k, j)\right.$ ) for any $l \geq k$, since $S\left(\bigcup_{j \geq i} \mathscr{B}(n, k, j)\right)_{i \geq k}$ is decreasing). Again by one of Burkholder's theorems ([2], p. 1196, Theorem 3 ) the upper envelope 


$$
\mathscr{B}(n):=S\left(\bigcup_{k=n}^{\infty} \mathscr{B}(n, k)\right)
$$

is sufficient for $\mathscr{P}_{n}$. Finally observe that $(\mathscr{B}(n))_{n \in \mathbf{N}}$ is a decreasing sequence. To prove this let $n \in \mathbf{N} . \mathscr{P}_{n} \subset \mathscr{P}_{n+1}$ implies $\mathscr{N}\left(\mathscr{P}_{n}\right) \supset \mathscr{N}\left(\mathscr{P}_{n+1}\right)$. This yields

$$
\mathscr{B}(n, k, m) \supset \mathscr{B}(n+1, k, m) \quad \forall k \geq n+1, m \geq k,
$$

and it follows immediately that

$$
\mathscr{B}(n, k) \supset \mathscr{B}(n+1, k) \quad \forall k \geq n+1 .
$$

This implies

$$
\mathscr{B}(n)=S\left(\bigcup_{k=n+1}^{\infty} \mathscr{B}(n, k)\right) \supset S\left(\bigcup_{k=n+1}^{\infty} \mathscr{B}(n+1, k)\right)=\mathscr{B}(n+1),
$$

where the first equality is valid since $\mathscr{B}(n, k)_{k \geq n}$ is increasing. Now $\mathscr{B}_{*}=\bigcap_{n=1}^{\infty} \mathscr{B}(n)$ is sufficient for $\mathscr{P}_{n}$ and every $n \in \mathbf{N}$ (again [2], p. 1196, Theorem 3). Lemma 1 yields the sufficiency of $\mathscr{B}_{*}$ for $(X, \mathscr{A}, \mathscr{P})$.

$\mathscr{B}_{\infty}$ contains $\mathscr{B}_{*}$, since $\mathscr{B}_{j}$ contains $\mathscr{B}(n, k, j)$ if $j \geq k \geq n$ and hence

$$
\mathscr{B}(n, k) \subset \bigcap_{i=k}^{\infty} S\left(\bigcup_{j=i}^{\infty} \mathscr{B}_{j}\right)=\mathscr{B}_{\infty} \quad \forall k \geq n .
$$

It follows immediately that $\mathscr{B}(n) \subset \mathscr{B}_{\infty}$ is valid, for all $n \in \mathbf{N}$, i.e. $\mathscr{B}_{*} \subset \mathscr{B}_{\infty}$.

REMARK 1. If the assumptions of Theorem 1 hold and in addition all sub- $\sigma$-fields $\mathscr{B}_{n}$ are necessary for $\left(X, \mathscr{A}, \mathscr{P}_{n}\right)$ (i.e. are $\mathscr{P}_{n}$-essentially included in any sub- $\sigma$-field which is sufficient for $\left.\left(X, \mathscr{A}, \mathscr{P}_{n}\right)\right)$ then the terminal $\sigma$-field $\mathscr{B}_{\infty}$ of the sequence $\left(\mathscr{B}_{n}\right)_{n \in \mathrm{N}}$ is necessary for $(X, \mathscr{A}, \mathscr{P})$. This follows immediately from

$$
S\left(\bigcup_{n=m}^{\infty} \mathscr{B}_{m}\right) \subset \mathscr{C} \quad\left[\mathscr{P}_{n}\right],
$$

which is valid for any sufficient sub- $\sigma$-field $\mathscr{C}$ of $\mathscr{A}$ and any $n \in \mathbf{N}$. Hence it may be deduced from Theorem 1 that minimalsufficiency of $\mathscr{B}_{n}$ for $\left(X, \mathscr{A}, \mathscr{P}_{n}\right), n \in \mathbf{N}$, implies minimalsufficiency of $\mathscr{B}_{\infty}$. This result is due to D. Landers ([4], p. 202, Theorem 7).

We now give a reformulation of Theorem 1 in terms of weak Blackwell-sufficiency. This is a weak version of a notion of sufficiency which has been introduced by $D$. Blackwell in [1].

DEFINITION 1. Let $(X, \mathscr{A}, \mathscr{P})$ be a statistical experiment.

1. Let $(\Delta, \mathscr{D})$ be a measurable space. A $\mathscr{P}$-weak Markov kernel from $(X, \mathscr{A})$ into $(\triangle, \mathscr{D})$ is a function $K: X \times \mathscr{D} \rightarrow \mathbf{R}$ such that

(a) $\forall D \in \mathscr{D}: K(x, D) \geq 0[\mathscr{P}]$, 
(b) $K(x, \Delta)=1[\mathscr{P}]$,

(c) for all sequences $\left(D_{n}\right)_{n \in \mathrm{N}}$ of pairwise disjoint $\mathscr{D}$-measurable subsets of $\Delta$

$$
K\left(x, \sum_{n=1}^{\infty} D_{n}\right)=\sum_{n=1}^{\infty} K\left(x, D_{n}\right) \quad[\mathscr{P}]
$$

holds true,

(d) $\forall D \in \mathscr{D}: x \mapsto K(x, D)$ is $\mathscr{A}$-measurable.

2. A sub- $\sigma$-field $\mathscr{B}$ of $\mathscr{A}$ is called weakly Blackwell sufficient if there is a $\mathscr{P}$-weak Markov kernel $K$ from $(X, \mathscr{B})$ into $(X, \mathscr{A})$ such that

$$
\int P(d x) K(x, A)=P(A) \quad \forall A \in \mathscr{A}, P \in \mathscr{P} .
$$

THEOREM 2. Let $(X, \mathscr{A}, \mathscr{P})$ be a statistical experiment and $\mathscr{B}$ be a sub- $\sigma$-field of $\mathscr{A}$. Then

$\mathscr{B}$ is weakly Blackwell sufficient

$\Leftrightarrow \mathscr{B}$ contains a sub- $\sigma$-field which is sufficient for $(X, \mathscr{A}, \mathscr{P})$.

Proof. This is shown in [3].

COROllaRY 1. Let $(X, \mathscr{A})$ be a measurable space, $\left(\mathscr{P}_{n}\right)_{n \in \mathbf{N}}$ an increasing sequence of nonempty classes of probability measures. For every $n \in \mathbf{N}$ let $\mathscr{B}_{n}$ be a sub- $\sigma$-field which is weakly Blackwell sufficient for $\mathscr{P}_{n}$. Then the terminal $\sigma$-field $\mathscr{B}_{\infty}:=\bigcap_{n=1}^{\infty} S\left(\bigcup_{m=n}^{\infty} \mathscr{B}_{m}\right)$ is weakly Blackwell sufficient for $\mathscr{P}:=\bigcup_{n=1}^{\infty} \mathscr{P}_{n}$.

Proof. This follows immediately from Theorem 1 and Theorem 2.

\section{References}

[1] D. Blackwell, Comparison of experiments, Proc. Sec. Berkeley Symp. on Math. Stat. and Prob. (1951), 93-102.

[2] D. L. BURKHOLDER, Sufficiency in the undominated case, Ann. Math. Stat. 32 (1961), 1191-1200.

[3] J. Hille, Über Suffizienz, Blackwell-Suffizienz und Bayes-Suffizienz, Doktorarbeit, Westfälische Wilhelms Univ. Münster (1995).

[4] D. LANDERS, Sufficient and minimal sufficient $\sigma$-fields, Z. Wahrscheinlichkeitstheorie und Verw. Gebiete 23 (1972), 197-207.

[ 5 ] J. Pfanzagl, Parametric Statistical Theory, de Druyter (1994).

Present Address:

INSTITUT FÜR MATHEMATISCHE STATISTIK, UNIVERSITÄT MÜNSTER,

EINSTEINSTRABE 62, D-48149 MÜNSTER. 